\title{
Analysis of 3-dimensional finite element after reconstruction of impaired ankle deltoid ligament
}

\author{
YUNHAN JI, XIANZHONG TANG, YIFAN LI, WEI XU and WENJUN QIU \\ Department of Orthopedics, Tongren Hospital Affiliated to Shanghai Jiaotong University School of Medicine, \\ Shanghai 200336, P.R. China
}

Received April 18, 2016; Accepted September 30, 2016

DOI: 10.3892/etm.2016.3894

\begin{abstract}
We compared four repair techniques for impaired ankle ligament deltoideum, namely Wiltberger, Deland, Kitaoka and Hintermann using a 3-dimensional finite element. We built an ankle ligament deltoideum model, including six pieces of bone structures, gristles and main ligaments around the ankle. After testing the model, we built an impaired ligament deltoideum model plus four reconstruction models. Subsequently, different levels of force on ankles with different flexion were imposed and ankle biomechanics were compared. In the course of bending, from plantar flexion $20^{\circ}$ to back flexion $20^{\circ}$, the extortion of talus decreased while the eversion increased. Four reconstruction models failed to bring back the impaired ankle to normal, with an obvious increase of extortion and eversion. The Kitaoka technique was useful to reduce the extortion angle in a consequential manner. Compared with the other three techniques, the Kitaoka technique produced better results for extortion angle and the difference was statistically significant. However, in case of eversion, there was no significant difference among the four techniques $(\mathrm{P}>0.05)$. Lateral ligament's stress in all the four models was different from the normal one. When the ankle was imposed with extortion moment of force, stress of anterior talofibular ligament with the Kitaoka reconstruction method was close to that of the complete deltoid ligament. When ankle was imposed with eversion moment of force, stress of anterior talofibular ligament with Kitaoka and Deland reconstruction methods were close to that of the complete deltoid ligament. We concluded that Kitaoka and Deland tendon reconstruction technique could recover impaired ankle deltoid ligament and re-established its normal biomechanics characteristics.
\end{abstract}

Correspondence to: Dr Xianzhong Tang, Department of Orthopedics, Tongren Hospital Affiliated to Shanghai Jiaotong University School of Medicine, 1111 Xianxia Road, Shanghai 200336, P.R. China

E-mail: xianzhong_tang1@163.com

Key words: 3-dimensional finite element, ankle, impaired deltoid ligament, Wiltberger, Deland, Kitaoka, Hintermann

\section{Introduction}

Independent inside ankle deltoid ligament injury is a rare type of injury (1). Most of these injuries are associated with malleolus medialis fracture and wither joint impairment. Among patients suffering from disability of later shin tendon, joint fusion of ankle and ankle replacement we can also see independent impaired deltoid ligaments (2).

There are three types of common inside ligamentous injury mechanisms: Ankle pronation-abduction, pronation-extorsion and supination-extorsion (3). Patients with unstable rotation need reconstruction and there are essentially four techniques available for reconstructing the chronically failed deltoid ligament. These are: i) Wiltberger; ii) Deland; iii) Kitaoka; and iv) Hintermann techniques (4). Due to the difficulty of model construction in animal ankle, clinical research usually fails to achieve a profound understanding on biomechanics and FEA (5).

In the present study, we applied 3-dimensional finite elements to compare the efficiency of these techniques in reconstructing deltoid ligament injuries of the ankle joint.

\section{Materials and methods}

Establishment of finite element model. We enrolled a healthy male volunteer (28 years of age, $172 \mathrm{~cm}$ and $65 \mathrm{~kg}$ ) without any trauma, inflammation or cancer for the present study. His ankle joint was examined under X-ray and using a 64-slice dual source computed tomography (CT) (Siemens Medical Solutions, Munich, Germany) his right ankle joint was scanned. MRI images were entered into the medical simulation software MIMICS 10.01 (Materialise, Leuven, Belgium). Images were divided according to the corresponding gray threshold in the MIMICS (the CT value of bones and soft tissue was different). After the border reading and the marrow cavity filling, the 3D model of the bones and cartilages of the ankle was generated. Each model was entered into software MAGICS 9.9, which was incidental to MIMICS software and was grid partitioned. Some irregular surface structures were repaired and smoothed. The final step was to enter the bone and cartilage models into the ANSYS 12.0 (Swanson Inc., Houston, PA, USA). Ligamentous structure was generated with the aid of ANSYS (set unit type, real constants and material property) and the finite element model 

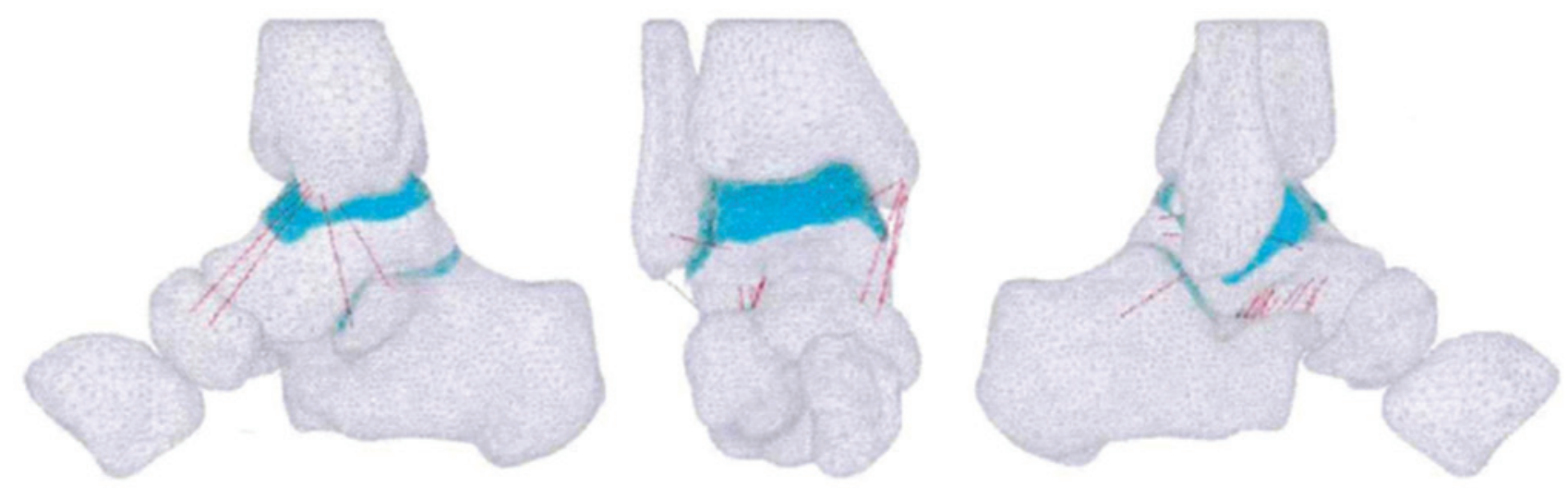

Figure 1. Ankle deltoid ligament 3-dimensional finite element model.

was generated (the total number of the overall transfer units were 48,600).

The bone surface structure of the units was partitioned by the rigid surface. The arthroidal cartilages were partitioned by the transformable 3D-tetrahedron. Ligament structure was simulated by bar unit which was tensioned only when the start and endpoints were confirmed according to the anatomy and CT image. Different ligaments were simulated by a different amount of bar units according to its length-width ratio. Articular cartilage was set to be the isotropic linear elastic material $(E=0.7 \mathrm{MPa}, v=0.49)$ and the simulation of the ligament around the ankle was defined by the following formula: $\mathrm{F}=\mathrm{A}\left(\mathrm{eB}^{*} \varepsilon-1\right)$ and parameters $\mathrm{A}$ and $\mathrm{B}$, where $\mathrm{F}$ referred to ligament stress, $\varepsilon$ was ligament strain and the parameter of ligament in the joint below was the same as the data for posterior talotibial ligament. The tendon transplantation was isotropic linear elastic material and the reconstruction needed four types of tendons: i) Posterior tibial; ii) plantaris; iii) peroneus longus; and iv) tendon of extensor pollicis longus. The initial cross-sectional area in each tendon was 48, 1.4, 37 and $2.91 \mathrm{~mm}^{2}$, respectively, and the elasticity modulus was 2,076, 1,172, 2,769 and $450 \mathrm{MPa}$, respectively.

Model-verified test. The model was carried out with anterior drawer test (ADT): We applied 50-150 $\mathrm{N}$ front draw force to the root bone, the flexion angle of the ankle joint was plantar flexion $20^{\circ}$ to dorsal flexure $20^{\circ}$. We calculated the predicted moving distance of talus. When the number of transfer units was more than 46,000 , the rate of convergence was below $0.45 \%$.

The sensitivity test applied to the material data of the anterior talofibular ligament was as follows: The original parameters of the anterior talofibular ligament in the model were $A=7.18$ and $B=12.50$. We immobilized $B$ to 12.50 at first and then $\mathrm{A}$ was changed by $0.1 \mathrm{~A}$ each time (6.4,7.1,7.9 and 8.6) while calculation of the change in the maximum forward displacement of talus was $0.5 \mathrm{~mm}$. While forward tension was $150 \mathrm{~N}$, we kept A unchanged and changed B by $0.1 \mathrm{~B}$ each time $(11.2,12.5,15$ and 16.2). Forward displacement of talus was changed by $1.0 \mathrm{~mm}$ at the most (forward tension was $150 \mathrm{~N}$ ). To further confirm the effect of the ligament pretension on the model, the ligament pretension of anterior talofibular ligament was adjusted to $110 \%$ and forward displacement of talus decreased by $22 \%$ under the tension of $150 \mathrm{~N}$. When adjusted to $90 \%$, the forward displacement of talus increased by $42 \%$ under the same tension.

Test method and observational index. The test was carried out in two steps: i) Talus and root bone were fixed and flexion angle of tibiofibula bucking was set at $-20^{\circ},-10^{\circ}, 0^{\circ}, 10^{\circ}$ and $20^{\circ}$; and ii) we fixed the tibiofibular and exerted extorsion or eversion torque to the root bone and the extorsion and eversion torque were set to 1.7 and $3.4 \mathrm{~nm}$. Initial stress of the ligament and tendon in the neutral position was 0 . The root bone was allowed to have degrees of freedom in five directions (forwardbackward translation, medial-lateral translation, near-far translation, intorsion-extorsion and inversion-eversion). When the model was operational, we intercepted the horizontal view to calculate the extorsion angle of the talus. We intercepted the anteroposterior view to calculate the eversion angle of the talus and each angle was tested three times by three investigators and the average values were calculated.

Statistical analysis. SPSS 19.0 statistical software (Chicago, IL, USA) was used for statistical analysis and data were expressed as mean \pm standard deviation. Comparison between groups was done with single factor ANOVA analysis. The enumeration data were expressed as a percentage. The comparison between groups was conducted using $\chi^{2}$ test. $\mathrm{P}<0.05$ was considered to indicate a statistically significant difference.

\section{Results}

Test model. Ankle deltoid ligament 3-dimensional finite element model (Fig. 1) and 27 link units were used to simulate the ligament structure. Complete injury of deltoid ligament model eradicated all deltoid ligaments from the model. The Wiltberger reconstruction model vertically cut the tendon. The near-end of tendon was connected to malleolus medialis while the far-end was connected to the nut bone. The Deland reconstruction model connected the near end of the transplanted peroneus longus tendon to malleolus medialis while the far-end connected to the medial cuneiform bone. Hintermann reconstruction model connected the far-end of transplanted plantaris tendon to tubercle of the nut bone while the near end was connected to malleolus medialis. Kitaoka connected the near end of transplanted hallucis longus tendon to malleolus medialis while the far end connected to entocuneiform (Fig. 2). 


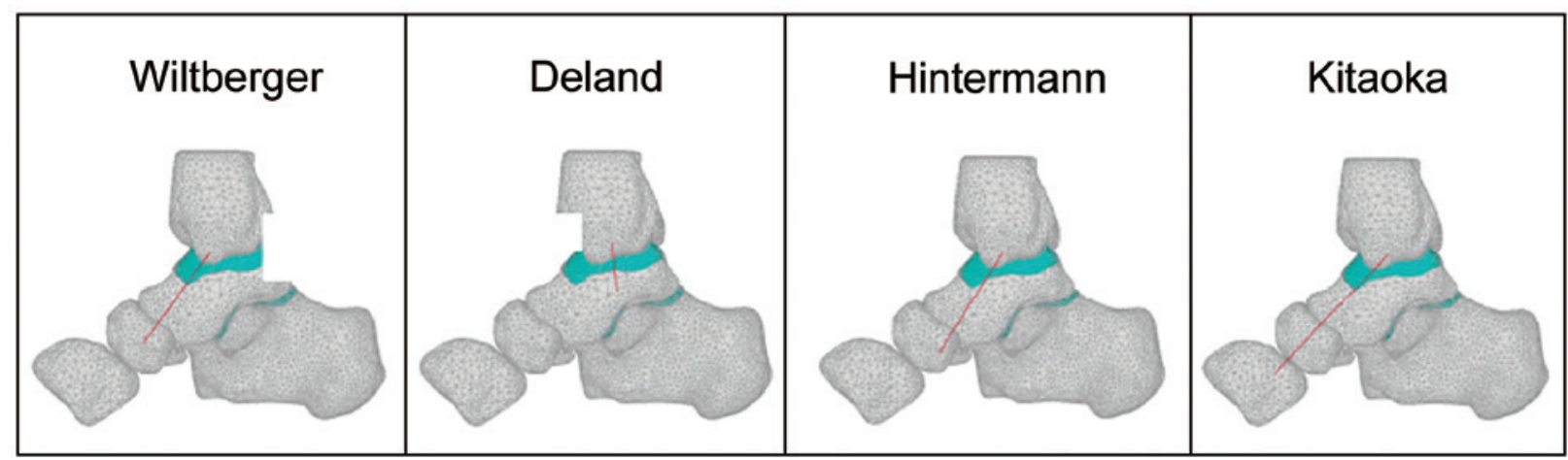

Figure 2. Injured deltoid ligament of ankle reconstruction 3-dimensional finite element models (successively are Wiltberger, Deland, Hintermann and Kitaoka).

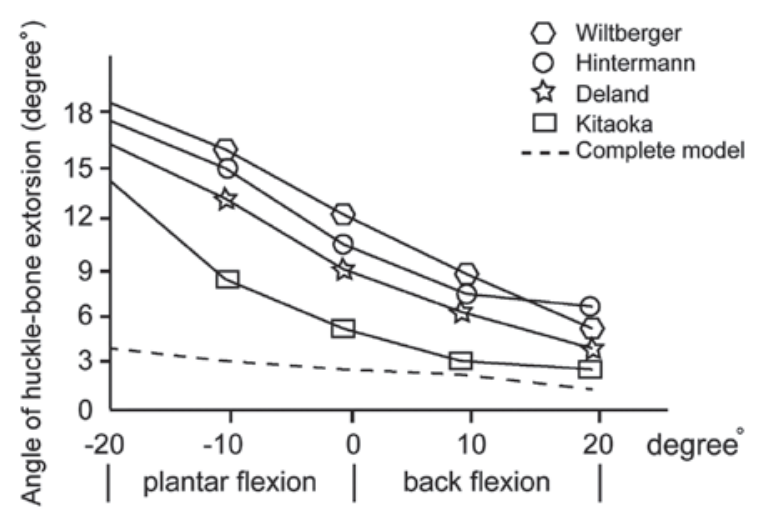

Figure 3. Angle of huckle-bone extorsion.

Angle of huckle-bone extorsion and eversion. In the course of bending, from plantar flexion $20^{\circ}$ to back flexion $20^{\circ}$, the extortion of talus decreased while the eversion increased. In the complete model of deltoid ligamentis in neutral position the average angle of huckle-bone extorsion was $3.6 \pm 0.7^{\circ}$ and the eversion angle was $4.2 \pm 0.5^{\circ}$. All four reconstruction models failed to fix the impaired ankle completely and there was an obvious increase in extortion and ectropion. Kitaoka helped to decrease the extortion angle more than the other three techniques and the difference had statistical significance $(\mathrm{P}<0.05)$. No statistically significant differences were found in the case of ectropion (Figs. 3 and 4).

Ligament stress analysis of the reconstruction model. The lateral ligament stress of four models was different from the normal one. When ankle was imposed with extortion moment of force, stress of anterior talofibular ligament with Kitaoka reconstruction method was close to that of the complete deltoid ligament. When ankle was imposed with eversion moment of force, stress of anterior talofibular ligament with Kitaoka and Deland reconstruction methods were close to that of the complete deltoid ligament (Fig. 5).

\section{Discussion}

Wiltberger techniques mainly reconstruct the shin ligament in the fascia colli superficialis of deltoid ligament instead of ligament of shin heel, anterior tibial ligament and posterior tibial ligament by means of transplanting the shin back

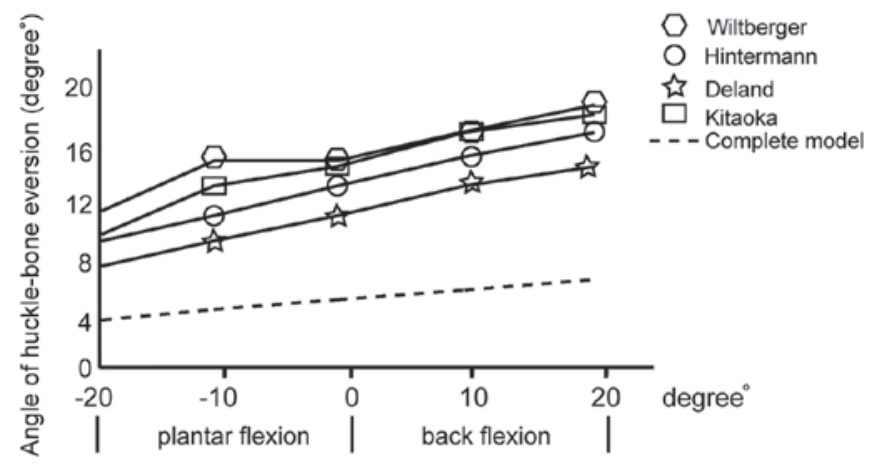

Figure 4. Angle of huckle-bone eversion.

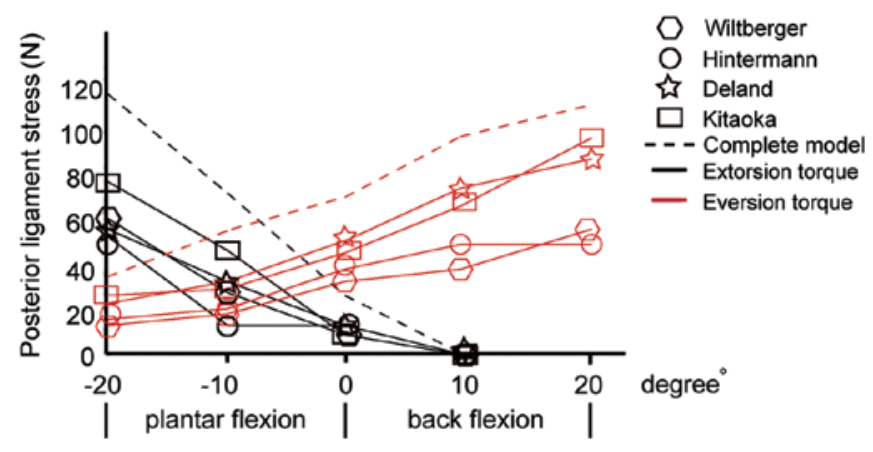

Figure 5. Posterior ligament stress analysis.

ligament. The entheses of ligamenta tibionavicular are medial malleolus and tuberositas ossis navicularis, from upper back to down front. When the ankle extorts, the displacement of the nut bone is obviously larger than the huckle-bone. Ligamenta tibionaviculare is passively extended then generates obvious force against extortion which maintains the stability of the ankle extortion. Both front and back ligaments play a crucial role in maintaining the stability of the ankle extortion. Therefore, Wiltberger can just partly recover the stability of ankle extortion (6). Hintermann mainly reconstructed shin ligament by transplanting plantaris muscle tendon instead of ligament of shin heel, anterior tibial ligament and posterior tibial ligament. The main difference between these techniques is not in the treatment results, but in the material used and the error occurs during the process (7). As for the Deland techniques, its entheses are the inside ankle and calcaneus. 
Line feed is roughly the same as long axis of the body. When the ankle extorts outward, the displacement of calcaneus is obviously larger than that of the huckle-bone. Ligament of shin heel is passively extended then generates obvious force against eversion which maintains the stability of the ankle eversion. However, the extortion circumference ratio of calcaneus is smaller than that of huckle-bone and its displacement is also smaller than that of the huckle-bone. Moreover, shin and ligament are in vertical position against each other which implies that Deland technique performs poorly against the ankle extortion (8). Kitaoka mainly reconstructed the shin ligament of deltoid ligament instead of ligament of shin heel, front and back by means of transplanting the hallux longus tendon. Compared with the Wiltberger and Hintermann techniques, the main difference is in its starting point and terminal point, respectively, the inside ankle and the inside entocuneifor. When the ankle extorts, the circumference of entocuneiform is obviously larger than that of the hucklebone and nut bone. Hence, Kitaoka techniques can generate significant moment against extortion and maintain the stability of the ankle extortion, but cannot recover the impaired ankle completely (9).

By constructing a 3-dimensional finite element model of normal ankle and analyzing the impairment reconstruction, we realized that all four techniques failed to fully recover the impaired ankle. These techniques could only reconstruct a part of the ligaments which could be useful to maintain the stability of extortion and eversion. Kitaoka technique obviously decreased the extortion angle which was related to Kitaoka's enthesis (10). As for eversion angle, there was no distinct difference among the four techniques. Our results revealed that Deland technique performed much better in terms of stability of eversion and this was because of the structure of the model (11). A few strategies were adopted: i) The material of ligament, bone structure and cartilaginous were mostly referred to pertinent literature therefore there may be a rather large bias (12); ii) the ligament material characteristic was defined as nonlinearity superelasticity (13); and iii) ankle joint was being fixed, but during the process of eversion, the movement of ankle joint was crucial (14). The stress of lateral ligament in the four techniques was different from the normal one. When the ankle was forced by extortion, the stress of anterior talofibular ligament was close to that of a complete one, and when the ankle was forced by eversion, the stress of posterior talofibular ligament was close to that of a complete one.

In conclusion, Kitaoka and Deland techniques performed better in impaired ankle deltoid ligament and could largely recover its biomechanical characteristics. However, further verification with larger clinical samples and control are needed.

\section{References}

1. Warner SJ, Garner MR, Hinds RM, Helfet DL and Lorich DG: Correlation between the Lauge-Hansen classification and ligament injuries in ankle fractures. J Orthop Trauma 29: 574-578, 2015.

2. Jung HG, Park JT, Eom JS, Jung MG and Lee DO: Reconstruction of superficial deltoid ligaments with allograft tendons in medial ankle instability: a technical report. Injury 47: 780-783, 2016.

3. Wang H, Gu Z, Liu Y, Xu J, Jan J, Zhang J and Peng C: Effectiveness of surgery in treatment of ankle fractrures associated with deltoid ligament injury. Zhongguo Xiu Fu Chong Jian Wai Ke Za Zhi 29: 416-419, 2015 (In Chinese).

4. Deland JT, de Asla RJ and Segal A: Reconstruction of the chronically failed deltoid ligament: a new technique. Foot Ankle Int 25: 795-799, 2004.

5. Xu C, Zhang MY, Lei GH, Zhang C, Gao SG, Ting W and Li KH: Biomechanical evaluation of tenodesis reconstruction in ankle with deltoid ligament deficiency: a finite element analysis. Knee Surg Sports Traumatol Arthrosc 20: 1854-1862, 2012.

6. Clanton TO, Williams BT, James EW, Campbell KJ, Rasmussen MT, Haytmanek CT, Wijdicks CA and LaPrade RF: Radiographic identification of the deltoid ligament complex of the medial ankle. Am J Sports Med 43: 2753-2762, 2015.

7. Hintermann B, Valderrabano V and Kundert HP: Lengthening of the lateral column and reconstruction of the medial soft tissue for treatment of acquired flatfoot deformity associated with insufficiency of the posterior tibial tendon. Foot Ankle Int 20: 622-629, 1999.

8. Chun KY, Choi YS, Lee SH, Kim JS, Young KW, Jeong MS and Kim DJ: Deltoid ligament and tibiofibular syndesmosis injury in chronic lateral ankle instability: magnetic resonance imaging evaluation at $3 \mathrm{~T}$ and comparison with arthroscopy. Korean J Radiol 16: 1096-1103, 2015.

9. Kitaoka HB, Luo ZP and An KN: Reconstruction operations for acquired flatfoot: biomechanical evaluation. Foot Ankle Int 19: 203-207, 1998.

10. Schottel PC, Baxter J, Gilbert S, Garner MR and Lorich DG: Anatomic ligament repair restores ankle and syndesmotic rotational stability as much as syndesmotic screw fixation. J Orthop Trauma 30: e36-e40, 2016.

11. Hogan MV, Dare DM and Deland JT: Is deltoid and lateral ligament reconstruction necessary in varus and valgus ankle osteoarthritis, and how should these procedures be performed? Foot Ankle Clin 18: 517-527, 2013.

12. Perrier A, Bucki M, Luboz V, Vuillerme N and Payan Y: 3D musculoskeletal finite element analysis of the foot kinematics under muscle activation with and without ankle arthrodesis. Comput Methods Biomech Biomed Engin 14: 1-2, 2015.

13. Liu Q, Zhao G, Yu B, Ma J, Li Z and Zhang K: Effects of inferior tibiofibular syndesmosis injury and screw stabilization on motion of the ankle: a finite element study. Knee Surg Sports Traumatol Arthrosc 24: 1228-1235, 2014.

14. Jay Elliot B, Gundapaneni D and Goswami T: Finite element analysis of stress and wear characterization in total ankle replacements. J Mech Behav Biomed Mater 34: 134-145, 2014. 